WellBeing International

WBI Studies Repository

$11-15-2002$

\title{
Modern elements of informed consent for general veterinary practitioners
}

\author{
Martin J. Fettman \\ Colorado State University \\ Bernard Rollin \\ Colorado State University, bernard.rollin@colostate.edu
}

Follow this and additional works at: https://www.wellbeingintlstudiesrepository.org/proveth

Part of the Animal Studies Commons, Bioethics and Medical Ethics Commons, and the Other Veterinary Medicine Commons

\section{Recommended Citation}

Fettman, M. J., \& Rollin, B. E. (2002). Modern elements of informed consent for general veterinary practitioners. Journal of the American Veterinary Medical Association, 221(10), 1386-1393.

https://doi.org/10.2460/javma.2002.221.1386

This material is brought to you for free and open access by WellBeing International. It has been accepted for inclusion by an authorized administrator of the WBI Studies Repository. For more information, please contact wbisr-info@wellbeingintl.org.

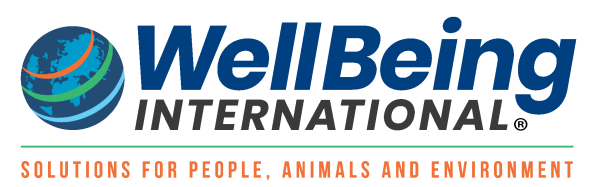




\title{
Commentary
}

\section{Modern elements of informed consent for general veterinary practitioners}

\author{
Martin J. Fettman, DVM, PhD, DACVP, and Bernard E. Rollin, PhD
}

B ecause veterinary medicine was historically ancillary to agriculture, informed consent was founded predominantly in preserving the owner's economic value of the animal undergoing diagnosis and treatment by the veterinarian. Accordingly, the principle interest of the animal owner was to protect his/her investment in a business asset, and the main concern of the veterinarian was to provide a reasonable estimate of the cost for medical services with a mind to minimizing expense and maintaining profitability for the client. The animal's interests in this business transaction were moot, and the relationship between animal and owner were overwhelmingly utilitarian (this broad claim was somewhat mitigated by the ethic of husbandry, wherein for certain cases more might be spent on treating an ill animal than it was worth). While this pragmatic relationship continues to be valid for many segments of production agriculture today, the growing populations of companion animals and the shift in veterinary-client relationships to one founded in the human-animal bond has effectively altered the paradigm of informed consent, even if the profession has not yet fully acclimated to this important change. In this instance, the economic foundation of informed consent has largely been replaced by an emotional and moral one, wherein risk and benefit are judged in terms of quality of life, empathy, anthropomorphism, and considerations for informed consent not unlike those for parent, child, and pediatrician.

The purpose of this article will be to provide some guidance to practitioners regarding the elements of informed consent as it applies to private practice with companion animals, and to stimulate thought about what constitutes effective communication between a veterinarian and client regarding benefits and risks associated with companion animal disease prevention and treatment. Informed consent for research purposes will not be considered because of the additional considerations of third-party beneficiaries of the agreement, in the form of researchers who receive recognition for publication of the results, companies that receive monetary benefit from the sale of experimentally proven therapies, and other animals that may ben-

From the Departments of Pathobiology, College of Veterinary Medicine and Biomedical Sciences (Fettman), and Philosophy

(Rollin), Colorado State University, Fort Collins, CO 80523-1601. Address correspondence to Dr. Fettman. efit in the future from the consent granted by the owner for risk assumed by his/her animal. Informed consent for production animal purposes will not be considered because of the overriding effect of economic motivation, even as it relates to the influence of animal welfare concerns on profitability.

\section{Elements Constituting Adequate Informed Consent}

Legal requirements-The basis for informed consent in medicine is to prevent patients from being treated against their will or the will of their guardians. In veterinary medicine, this principle leads to the objective that owners be provided adequate information so they can make the right decision for their pet and for themselves. Ernst and Cohen ${ }^{1}$ made an important point when they said, "The completion of a standard consent form does not, however, constitute consent itself; it is merely evidence that consent has been given." It is the exchange between owner and veterinarian of the information necessary for informed consent that constitutes the real test of whether a concerted effort has been made at effective communication and understanding.

The veterinary literature includes some discussion of informed consent, although it is primarily directed at legal considerations, and not moral ones. Our discussion will attempt to embrace both concerns. Wilson et $\mathrm{al}^{2}$ offered three tests for informed consent. The first option is to disclose facts that a reasonable medical practitioner in a similar community and of the same school of medical thought would have disclosed regarding the proposed treatment. The second option, described as the objective minority view, is to disclose all risks that would be material to a reasonable, prudent person in the client's position. The third option, described as the subjective minority view, is a full disclosure standard that applies to risks associated with a client's decision with respect to treatment received or not received.

Hannah $^{3}$ has stated that clients have a right to know the diagnosis, proposed treatment, and potential risks associated with a veterinarian's management of their animal(s). Clients may vary in their ability to understand the explanation, and veterinarians may vary in their knowledge and ability to communicate that information, so a reasonable consent form is recommended. Examples of items for the informed consent that Hannah gave included information about 
after care (feeding, medication, return visits, or any items that are appropriate in view of the condition of the animal), information about medications (instructions should be thorough about the amount, frequency and method of medicating, and the procurement and storage of the drugs or other materials to be used), information about drugs (information should be thorough, and probable adverse effects should be explained), failure to warn about the security for prescription drugs for purposes other than those defined by the veterinarian vis-à-vis safety of family members and other pets, failure to warn and instruct when the client assists (to avoid injuries that might be probable in the particular circumstance), and informing prospective purchasers of communicable disease. Although this information is technically comprehensive, it is not all-inclusive with respect to conceptual principles we will elucidate herein.

Medical requirements-Although it is not necessary or feasible to conduct a minicourse on the technical subjects relating to an individual patient, a good faith effort should be made to include the following information: communicate the medical nature of the problems, explain the diagnostic tests used to evaluate the problems and to prioritize diagnoses, discuss the relative risks and benefits of additional diagnostic procedures or implementation of specific treatments, describe how response to treatment will be assessed to identify recovery and to recognize warning signs of treatment failure, worsening of the condition, or development of adverse effects, and provide a reasonable prognosis for recovery or recurrence.

Risk/benefit discussion-No client expects every ill patient to be completely cured following treatment by a veterinarian. However, few clients expect their animal's condition to worsen following veterinary care, especially for diseases that are commonly viewed as being self-limiting or nonfatal. The relative probability for a patient with a given diagnosis to respond to a particular course of management, without undue adverse effects, must be communicated to the client. Each potential risk for treating or not treating problems should be discussed. Likewise, the definition of response to treatment must be clear so that the client understands the veterinarian's concept of remission or cure, potential return of function, or loss of function after treatment.

For example, a veterinarian might recommend surgical reduction to treat an otherwise healthy young dog's aural hematoma. This would involve general anesthesia, one or more skin incisions, removal of the blood clot, implantation of a drain, and obliteration of the space with a series of sutures through the earflap. ${ }^{4}$ Alternatively, one might recommend sedation with local anesthesia and percutaneous aspiration, with or without injection of a corticosteroid. ${ }^{4}$ The former is more costly and incurs some anesthetic risk, but is more likely to result in uncomplicated recovery from the problem. The latter may present greater risk for recurrence and infection. Nevertheless, the relative benefits and potential costs of both options should be explained to the owner. Changing a single variable in this example can vastly alter the relative balance of benefit and risk. For instance, if the aural hematoma developed in a 12-year-old obese Basset Hound for whom anesthetic risk is deemed considerable, the decision regarding surgical treatment might be very different from that for a 2-year-old lean healthy Labrador Retriever. The relative benefits and potential costs of both options should once again be presented to the owner.

Interactive risks-Interactive risk entails the likelihood of more than one risk factor combining in effect to induce greater than expected problems - the whole is greater than the sum of the parts. There may be interactive risks for not treating one or more disease problems. For example, inadequate control of pain may interact with other disease signs to induce a greater degree of anorexia and stress that subsequently impairs wound healing. There may be interactive risks for combined effects of treatments used. For example, interactions among diet and drugs used to treat separate but concurrent disease signs may alter drug absorption, metabolism, or efficacy, and may even lead to drug toxicosis. ${ }^{5}$

In the example of an aural hematoma, interactive risk may include not detecting and treating underlying problems that may have led to an animal shaking its head and traumatizing the ear with the subsequent formation of a hematoma. Discomfort caused by implantation of a drain in the reduced hematoma space may lead to the interactive risk of additional head shaking and aural trauma that can be avoided by immobilizing the affected ear by bandaging it to the head. Identification of an underlying otitis externa may require treatment with antimicrobials, but the combination of pain and oral administration of antimicrobials may entail interactive risk for gastrointestinal upset, anorexia, and delayed wound healing.

Waivers-If a veterinarian makes a professional needs assessment for the management of a case, the client, as the animal's legal owner, must still be given the option to accept or reject that assessment. If more than one potential course of action is possible, the veterinarian may make his/her recommendation for the best course to follow, but it is still the owner's right to choose among those options. Thus, in addition to the need for informed consent and written documentation of that communication, it is also frequently advisable to have a signed waiver for specific items that may be rejected. Decisions to reject a veterinarian's advice for diagnostic tests or treatment modalities are often dependent on the cost of the proposed item. Patient advocacy connotes the concept that veterinarians should always recommend what they believe will be the best course of action for the patient. However, a signed waiver may be necessary when the owner chooses an option that is less demanding on their resources.

For example, if anesthesia and surgical correction are recommended for an aural hematoma, it is likely that preoperative laboratory screening tests would be recommended to determine potential risks for the procedure. If the affected animal is young and determined 
to be apparently healthy on the basis of physical examination, it is possible the owner may decline the complete preoperative laboratory tests and choose only some of the tests recommended by the veterinarian. If the veterinarian agrees to this level of screening, a waiver would be indicated to absolve him/her of legal responsibility should untoward events follow.

\section{To Whom is the Veterinarian's Primary Obligation and Does This Relationship Necessitate Informed Consent?}

Rollin ${ }^{6}$ once argued that veterinarians may adhere to two models of obligation. One is that of a garage mechanic wherein the veterinarian sees her/himself as operating only at the behest of the owner who might require a mechanic to junk a vehicle if repair costs exceed its value. In contrast, there is the pediatrician model wherein the veterinarian sees her/himself as operating predominantly on behalf of the patient and would not allow a parent to "junk" a child.

It is possible to consider our responsibility to one of three entities when treating an animal. The first is the animal itself. It is the animal that is ill and requires treatment or that may risk disease if appropriate preventive measures are not taken. Some refer to this relationship as one of patient advocacy, wherein regardless of the owner's stated preference for treatment, the veterinarian must do his/her best to advocate on behalf of the patient for the course of management that is most likely to provide the greatest benefit with the least risk, regardless of resource costs. An extreme view of patient advocacy might lead one to conclude that there is no need for informed consent from the owner, based on the assumption that the veterinarian knows what is best and need not explain his/her decision-making process in selecting treatment. In fact, there are situations where veterinarians are asked to serve only as a patient advocate, when an owner adamantly insists that anything be done for his/her beloved pet, regardless of the cost or inconvenience to the owner, as long as the animal does not suffer unnecessarily or without clear benefit. This scenario need not have resulted from the effect of Aesculapian authority (vide infra) on an owner's ability to make informed decisions. However, it would nevertheless be incorrect for the veterinarian to omit a discussion of informed consent in this case. There must be agreement at the outset as to the owner's expectations for improvement and acknowledgement of risk, however remote the possibility.

For the exemplar of a dog with an aural hematoma, the patient advocate might insist only on the option for surgical correction, preceded by appropriate preoperative laboratory screening, followed by necessary postoperative analgesic and antimicrobial treatment, as well as bandage changes to maintain cleanliness and immobilization of the affected ear. The owner would not be given the opportunity to reject complete treatment as recommended. Communication of potential risks and the expected course of recovery would nevertheless be included in adequate informed consent discussions.

Consider an example of a healthy young English
Springer Spaniel that experiences separation anxiety, manifested by vocalization and destructive activity while left home alone each day when its owners are at work. Many practitioners choose to handle nonaggressive behavioral problems without referral to behavior specialists. Today, patient advocacy may speak for referral of this dog to a behaviorist who may be able to more completely assess the factors contributing to the problem. Subsequent investigations may reveal the need for more outdoor activity for the dog, playtime with the owners or other animals, desensitization training to adapt to the emotive responses to its owners' departure each day for work, and reassurance through graduated departures that leaving may be for variable time periods that need not lead to endless hours of separation. ${ }^{7}$ Likewise, patient advocacy may indicate the need for behavioral modification of the owners in how they separate from their pet and how they greet it upon return. Many of the potentially indicated methods of managing this problem will involve considerable resource expense for the owners, but the prime objective is to respond to the patient's needs without concern for the attendant costs.

The second entity to consider is the animal owner, who is the legal guardian and will bear the financial burden, time, and labor costs of managing the outpatient. In this scenario, patient advocacy should not necessarily be abandoned, but the owner will have the responsibility of making the decision among alternate choices for diagnosis or treatment. If there is agreement between owner and veterinarian on the course to follow, the necessity for informed consent follows the same logic as that for patient advocacy, regardless of whether patient advocacy has driven the decision-making process. If there is disagreement because of conflicts between a veterinarian's patient advocacy and an owner's resource constraints, informed consent and signed waivers serve as cornerstones for the medical protection of the patient and legal protection of the veterinarian.

It is possible, in the example of the aural hematoma, that the parsimonious owner might choose percutaneous aspiration and teat cannula placement for drainage under sedation without placement of auricular sutures or the continued postoperative use of analgesics. An extreme option for the thrifty or financially deprived owner is also to do nothing, to accept the animal's discomfort and the risk of infection during the natural course of healing, and to be content with any degree of scarification that may result. Informed consent and appropriate waivers may be required to document these decisions, whether they conflict with the veterinarian's recommendations or not, if alternative preferred treatments such as surgery are not selected.

If one's concerns were predominantly for the owner's interests in resolving the example case of a pet with separation anxiety, behavioral modification with the consultation of a behavioral specialist might not be given the same priority as avoidance management and medication in the form of kenneling the misbehaving dog while the owners are gone or treating it with anxiolytic drugs to accelerate learned compliance.

The third entity offered for consideration by some 
practitioners is the human-animal bond. Some veterinarians believe that the bond is the direct object of moral attention for interactions among the owner, patient, and veterinarian. On a positive note, this may offer the veterinarian a means of coalescing divergent or convergent needs of the owner and animal. In other words, if the veterinarian's duty is to the bond, then patient advocacy, human resource limitations, and the emotional quandary created by their divergence can be addressed. Informed consent would include the risks and benefits of what the veterinarian, acting as a surrogate psychoanalyst, interprets to be the appropriate balance between medical necessity and resource expediency on behalf of the relationship between owner and animal. If this is consciously used as a basis for informed consent, the informed consent may become more complicated than delivery of medical fact and opinion because of the inclusion of some rationale based on effective two-way communication of bondrelated information. A skeptic could find that treating the bond and tailoring informed consent to the bond serves issues of the animal-owner relationship more deserving of detailed psychoanalysis. Is the bond a manifestation of misplaced guilt by the owner over perceived transgressions against the animal, displaced emotions from the owner over unsatisfactory human relationships, or replaced expression of the owner's self-esteem? The bond is an accepted object for attention by the veterinarian. However, she/he must undergo proper training and rehearsal of the interpersonal skills necessary to function successfully as an advocate of the bond.

For a young, otherwise healthy dog with a small aural hematoma, a veterinarian could infer that even if lesser methods might be expected to be sufficient, full surgical treatment entailing owner involvement in postoperative care of the wound, bandaging, and medication may serve the additional purpose of reinforcing the owner-animal relationship while avoiding risks for recurrence, however unlikely, should the lesser method have been chosen.

For the exemplar of an English Spring Spaniel with separation anxiety, primary consideration of the human-animal bond might have led to preemptive counseling for the owners on selection of an appropriate breed of dog for a pet who might better endure prolonged periods alone in a house. Responding to the threat posed to their bond by this behavioral problem might lead to a preference for training to reinforce the bond without medication that can have the adverse effect of personality changes that detract from the owner-pet relationship.

\section{Client Communications as an Essential Practice Management Tool}

Veterinarians, like most scientists, are highly trained in the cognitive skills necessary for medical practice. Clinical competence is often measured in terms of diagnostic accuracy and therapeutic success. Inadequate communication with the client may result in less than full appreciation of the difficulty and uncertainty involved in making a diagnosis, or disappointment in the outcome of treatment if the relative risks and benefits of that treatment are not discussed. Veterinarians and their staff must develop and practice the behavioral skills necessary for truly informed consent by their clients. In addition to medical knowledge and procedural skills, the ethical aspects of clinical practice require cultivation of trust between client and veterinarian through communication of veterinarian recommendations and client preferences.

$\mathrm{Kat}^{8}$ observed that the "doctrine of informed consent was not designed to serve as a blueprint for interactions between physicians and patients. At best, judges wanted to prod physicians to articulate medical standards of disclosure practices that would conform better with basic legal principles." Practitioners still seem to struggle with the value of informed consent in the delivery of quality medical services, above and beyond its role in legal protection.

An oncologist might reasonably view a disease-free interval of several months duration following chemotherapy for a particular cancer as a highly successful remission. However, a poorly informed owner may have expectations for a remission of years' duration. If the object of a veterinary oncologist's ministrations for cancer is the animal's well-being, then quality of life and remission from clinical signs or pain might be the only concern. If the object of treatment for cancer is the owner's desire to do what is best in a costeffective manner that will not incur additional discomfort for the animal, however briefly, no matter the potential survival time, management and informed consent would be different. Likewise, if the object of treatment is the bond itself, a very careful discussion between veterinarian and owner may be required to adequately communicate the importance of additional quality time for the owner-pet relationship for the owner to come to terms with issues of grief and anticipated loss. One could hypothesize that fully informed consent for a terminal disease in modern veterinary practice must also include some proactive counseling by the veterinarian for the owner's anticipated loss.

Consider the scenario of an owner who believes that his veterinarian verbalized the instruction that if "the owner doesn't pay for the recommended treatment, the veterinarian would have to kill his dog." However, the veterinarian actually said, "This is going to be a recurrent and expensive problem, and if you don't want to spend the money and invest your time in aftercare, then for the sake of the animal we should consider euthanatizing your pet." Needless to say, more emphasis should be placed on effective client communications in veterinary curricula so that misapprehensions such as this can be anticipated and avoided through careful selection of words and exchange of information necessary to adequate informed consent.

\section{Evidence-based Medical Practice}

Fontanarosa and Lundberg" said, "There is no alternative medicine. There is only scientifically proven, evidence-based medicine supported by solid data or unproven medicine, for which scientific evidence is lacking." Informed consent should include adequate information and discussion about the risks and benefits of all reasonable diagnostic and therapeu- 
tic options. It is in the definition of reasonable that reasonable people may differ. Ernst and Cohen ${ }^{1}$ suggested three elements to consider in this area; the probability of benefiting from the procedure, the probability of risk associated with the procedure, and the alternative options feasible and available, as well as their risk and benefits. They indicate that US courts have not typically included complementary and alternative medicine that are outside of consensus and conventional standards in the feasible and available mandate. However, this is undergoing continuous change as more human patients or animal owners request information on unconventional treatments, and as more experimental evidence is published regarding the potential benefits and risks of alternative practices.

Complementary and alternative medicineComplementary and alternative veterinary medical (CAVM) practices should be held to the same ethical and evidential standards as conventional medicine. Even if a veterinarian is disinclined to recommend CAVM approaches, the owner may request information regarding the comparative risks and benefits of CAVM compared with conventional management. It will likely become more common for clients to request some combination of complementary and conventional medical practices (integrative medicine) in the management of their animals, or to seek alternative approaches alone when traditional management has failed to induce a satisfactory result. Particularly for those clients whose animals suffer a chronic or life-threatening condition that will not be resolved by conventional medical treatment, complementary practices may appear to offer additional hope. However, it is the veterinarian's responsibility to distinguish between untested, unconventional treatments for which reasonable mechanisms of action may be intuitive, and those that are untestable because of incompatibility with known physical laws (eg, homeopathic remedies) or impossibility of designing placebo-controlled, doubleblind trials (eg, communicating with spirits of deceased animals).

Anecdotally accepted common practices-One could question the ethics of practitioners who recommend conventional diagnostic procedures or therapeutic plans for which no controlled research exists to support claims of efficacy and safety. Certainly, those who persist in advocating treatments that have been proven to be ineffective or even to cause harm cannot find protection in genuine informed consent. Much of veterinary medical practice is founded on historical data gathered under circumstances that would not pass the rigors of modern research requirements for the control of placebo effects on the part of the veterinarian, client, or animal. Thus, the principles underlying informed consent can apply equally to the communication of potential benefits and risks for conventional and alternative medical practices promulgated in the absence of experimentation that can pass critical review. This raises the important question of what should be accepted as an external measure of efficacy or safety, especially when controlled studies have not been published. Is it acceptable to recommend a treatment for which there are no supporting data if there are likewise no data questioning efficacy of safety? Is it acceptable to use anecdotal and poorly documented reports of success as justification for recommending a course of management? Can the recommendation for a treatment be based on unsubstantiated belief if, through informed consent, communication of the uncertainty inherent in this decision is satisfactorily attempted?

Informed consent for owner-initiated versus veterinarian-initiated consideration of unproven treatments-If an owner specifically requests information regarding CAVM treatments, the veterinarian is dutybound to provide the information she/he is aware of, including the surmised balance of risks and potential benefits. Fully informed consent for treatments the veterinarian is not fully familiar with may require referral to a more knowledgeable individual or another reputable source of information. Informed consent need not include comprehensive transfer of information from the veterinarian if a reasonable alternative source can be referenced. Informed consent need not stifle a veterinarian's expression of his/her own opinion regarding the values or dangers of nonconventional or anecdotally accepted, yet unproven, treatments. The practitioner should offer any information that she/he may deem useful to an owner's decision-making process, and the veterinarian-client relationship should be maintained if at all possible as a duty to the concept of patient advocacy when unconventional treatment is selected. A veterinarian should not abandon the patient if an owner makes a decision at odds with one's own beliefs, because there is still ample opportunity to protect the patient's health and to maximize the benefit to both owner and patient. ${ }^{10}$ Much like the pediatrician is in a position to balance a commitment to family-centered care with the ethical responsibility to guard the welfare of children, so is the veterinarian in a position to safeguard the well-being of the pet. ${ }^{10}$ Further, the veterinarian can help to establish clinical outcomes and target behaviors or signs of improvement or deterioration that can be observed and measured. ${ }^{10}$

It is possible for the veterinarian to initiate discussion of CAVM, much like the advocacy of anecdotally accepted treatments not yet subject to full scientific scrutiny. As a fundamental part of patient advocacy, it may be more useful in the long run to offer information on unconventional treatment modalities as part of informed consent discussions than to have avoided the topic and subsequently lose the owner-animal-veterinarian relationship. Some individuals believe that complementary and alternative practices offer only false hope to desperate owners of animals with nonresponsive diseases. If one believes in treating the bond, and the risk attendant to unconventional methods is small, the veterinarian-turned-psychoanalyst may have much to offer through discussion and implementation of alternative methods. However, financial dimensions of the unconventional practice should be considered if the veterinarian truly believes the modality is without medical value, to protect the client from being defrauded.

Standard of practice-Veterinarians are required to communicate to the client the generally accepted 
best course of management of their animal's condition, as well as the options that may exist for differences in cost and for relative risk and benefit. Once agreed on, the veterinarian is required to perform those diagnostic and treatment procedures with a level of skill comparable to most of his/her professional colleagues, and to recognize and promptly respond to any untoward reactions that a licensed veterinarian would generally be expected to be familiar with. To maintain an acceptable standard of practice, individual veterinarians must actively subscribe to a principle of lifelong learning and acceptance of change. Continuing education is only one facet of this, wherein the individual must continually assess which existing knowledge and skills to retain, which ones to discard, which ones to replace with new information or methods, and how best to evaluate their relative efficacy in his/her hands. For example, a veterinarian in practice today should be familiar with the various options for surgical correction of a ruptured cranial cruciate ligament. She/he may continue to be capable of performing an excellent ligament reconstruction procedure, but may choose to recommend referral to an orthopaedic specialist for a tibial plateau lateral osteotomy. The relative costs, benefits, and risks must be communicated to the owner for him/her to decide which option to choose. Despite strong opinions in favor of one procedure versus another, either is still acceptable under current standards of practice. Malpractice in cases like these may hinge more on inadequate communication of the contrasts between procedures than on the skill with which they are performed or even the outcome that results for the patient.

\section{Aesculapian Authority}

Rollin $^{11}$ described Aesculapian authority as "the uniquely powerful authority vested in those that society perceives as healers, historically traceable to the time when medicine was inseparable from magic and religion." The challenge for veterinarians is to provide enough information and recommendations to facilitate adequate informed consent from the owner without compromising the health of the patient, nor imposing personal preferences that may impose acceptance of a level of risk against the client's own inclinations. By no means does this require the veterinarian to maintain absolute neutrality when discussing options for diagnosis or treatment. To the contrary, patient advocacy requires that some preferences be expressed to the owner, for the sake of evaluating efficacy, potential risk, and resource requirements for implementation. There may also be times when patient advocacy will not support patient treatment, such as when resource limitations may impair the owner's ability to provide aftercare or to prevent recurrence. Certainly, recommendation for euthanasia may sometimes be indicated, even when resources are available, a course of management is evident, but the anticipated duration or magnitude of response may be limited. The owner will rely on a veterinarian's expertise to predict, based on past experiences, what course of action to follow. Neutrality cannot be used for cover from a difficult decision under the aegis of avoiding the influence of Aesculapian authori- ty. As an example, the veterinarian treating a cancer patient must be careful to synchronize his/her definitions of successful remission and prolonged survival with those of the owner, so that adverse effects of treatment and recurrence of the cancer will not become a surprise.

Patient advocacy-Veterinarians should avoid creating a situation where the owner will choose only against a course of action because of inadequate communication. Rather, veterinarians should try to present options of various risk and benefit relationships that will allow the owner to make a decision that will benefit the animal. This can sometimes be accomplished by shifting the owner's perception of what, according to Ubel "constitutes an omission and a commission" in the practitioner's recommendations. Because decision making is often driven by an aversion to making errors of commission that attach responsibility for blame, describing treatment options only as active courses of action can create a paradigm where the owner accepts some risk as a foregone conclusion of the process. However, when a veterinarian recommends euthanasia to an irresolute owner faced with the difficult decision of choosing against prolongation of his/her pet's life with a substantial reduction in quality of living, the veterinarian must also accept responsibility for later guilt-derived perceptions by the owner of a veterinarian-driven error of commission. Once again, fully informed consent in this situation must also include some proactive grief counseling to minimize the risk for owner misconception.

Consider the case example of a young, otherwise healthy horse with lameness caused by superficial digital tendonitis in the distal metacarpal region. Conventional medical treatment might include stall rest, gradually increased hand walking, nonsteroidal anti-inflammatory drugs, cold compresses, and even short acting corticosteroids. ${ }^{13}$ If this approach is not completely successful, surgical treatment may be indicated to include tendon splitting and even transection of the palmar annular ligament. ${ }^{13}$ However, if this, too, is not successful in restoring as much function and relief from discomfort as the owner had anticipated, she/he may express willingness to consider other therapies. Firing may have been recommended many years ago as a means of counter irritation to stimulate healing. Chiropractic manipulation or acupressure might be considered today as alternative therapies. Firing had been used in the past in the treatment of lameness in horses. Although counter irritation remains the basis for some treatments in veterinary and human medicine, there is no evidence of the efficacy of pin firing to justify the pain and tissue damage caused by the procedure, nor the risk for secondary infections or loss of function from scarring. Considerations of patient advocacy and standard of care should prompt the veterinarian to dissuade the owner from consideration of firing as an alternative. Although there are no controlled studies of the efficacy of chiropractic manipulation or acupressure for equine lameness, from the standpoint of patient advocacy, one could offer these alternative treatments because of anecdotal reports of 
efficacy without substantial risk for detrimental effects with appropriate informed consent.

White coat syndrome-In the context of patient decision making in human medicine, this is manifest as physician paternalism. In any context, the rights and privileges attendant to wearing a white laboratory coat have a psychological effect on the subject's decisionmaking ability and even a physiologic effect on the subject's behavior (eg, sympathetic nervous systemmediated changes in heart rate, blood pressure, respiratory rate, and depth). Practitioners must be careful not to overreact to the white coat syndrome and inadvertently withhold information or recommendations because of fear of unduly influencing the owner's decision for his/her animal.

Issues of cost-This may arise in the situation where the owner asks, "What would you do for your animal in this situation?" wherein she/he understands that a course of action is preferred because of overwhelming benefit and minimal risk, but the cost in terms of many resources (eg, money, owner time, animal discomfort) is viewed as prohibitive. It may also arise when the differences between treatment options are not large or are not adequately communicated by the veterinarian. Given the veterinarian's training and experience, she/he is likely more capable of making an informed decision, but cannot intuit intangible preferences that might dissuade an owner from the prescribed course of action.

\section{Certified Continuing Education}

There are considerable divisions within our profession regarding the necessity of required continuing education (CE) for maintaining licensure. There is little information regarding the efficacy of CE in general, much less for individual programs offered in various venues available to practitioners. Some practitioners view CE as an unnecessary burden to meet bureaucratic requirements for licensure. Others accept it as a method to enforce the principle of lifelong learning. Yet, others go through the motions of attending CE conferences without assimilating the instructional materials. Few CE programs clearly articulate the active or behavioral learning objectives expected of the participants, and some endorsing organizations have no objective criteria for certifying the instruction delivered.

One could make a case that the veterinarian who never makes a serious mistake will never have need for CE. However, documentation of CE provided by an acceptable certifying organization may become necessary when, despite a practitioner's best efforts at lifelong learning and client communication, untoward outcomes of a patient's management lead an owner to file malpractice charges or to seek legal recourse. Whether $\mathrm{CE}$ is required for licensure, legal action against the veterinarian will likely require some documentation of CE participation.

Practitioners should know, through CE, what the standard of practice is, whether they accept that notion themselves, or are in fact capable of delivering that treatment. For example, one may not believe that pain control is a fundamental necessity of all medical and surgical procedures that may inflict discomfort on the animal. However, the standard of care today, derived from federal law regulating pain control in laboratory animal research subjects, requires implementation of a valid method for pain control in any animal that experiences clinical signs or undergoes a treatment protocol that is likely to induce pain. Concepts of pain control may not have been adequately covered in veterinary school curricula in the past, but there are sufficient opportunities for continuing education now to address this issue more effectively. One cannot deliver adequate informed consent on this topic without adequate, recent instruction in this area.

If CE is truly effective at promoting learning cognitive and behavioral skills, it protects the animal. If $\mathrm{CE}$ is effective at facilitating the veterinarian's awareness and effective communication of current practices, it protects the owner. If $\mathrm{CE}$ is documented properly in accordance with licensure requirements, it protects the veterinarian.

Management-Continuing education directed at effective practice management, business administration, and client communications will promote effective informed consent.

Legal-Continuing education directed at understanding the pertinent state practice act, current case law, and the legal requirements for client communications and medical records documentation will promote effective informed consent.

Medical-The underlying principle of informed consent is, after all, patient advocacy; how to communicate, select, and conduct the best course of action for a diseased animal.

Moral-social ethics-Veterinarians must be familiar with the ever-evolving field of animal welfare. Beyond the duty to the individual animal for appropriate treatment, there is the duty to the owner to provide adequate counseling on treatment effects, and to other animals or people who may be affected by management practices, communicable diseases, environmental impacts, or trans-species patterns of abuse if not recognized and dealt with effectively.

\section{Referral-the Final Act of Informed Consent in Modern Veterinary Practice?}

Veterinarians are licensed to perform all aspects of medical and surgical procedures on animals. Yet, few general practitioners would attempt aspects of advanced practice more often conducted by board-certified specialists in particular disciplines, unless they themselves have received reasonable training and experience with the planned procedure. Thus, the final undertaking of informed consent, consistent with accepted standards of practice, may sometimes become the act of recommending referral of a difficult case to a more highly trained specialist. Academicians often observe that our greatest professional responsibility is to teach young veterinarians to objectively assess their capabilities and recognize limitations that should lead 
to consultation with a more experienced individual or actual case referral. Client gratitude for effective communication depends on truthful conveyance of information, even if the message is "I don't know."

\section{References}

1. Ernst E, Cohen MH. Informed consent in complementary and alternative medicine. Arch Int Med 2001;161:2288-2292.

2. Wilson JF, Rollin BE, Garbe JA. Professional liability. Chapter 6. In: Wilson JF, ed. Law and ethics of the veterinary profession. Yardley, Pa: Priority Press Ltd, 1993;143-145.

3. Hannah HW. When can failure to inform support a malpractice claim? J Am Vet Med Assoc 2001;218:1419-1420.

4. Fossum TW, Hedlund CS, Hulse DA, et al. Aural hematomas and traumatic lesions of the pinna. In: Fossum TW, ed. Small animal surgery. 2nd ed. St Louis: Mosby Year Book Inc, 2002;246-250.

5. Fettman MJ, Phillips RW. Dietary effects on drug metabolism. Chapter 27. In: Hand MS, Thatcher CD, Remillard RL, et al, eds. Small animal clinical nutrition. 4th ed. Marceline, Mo: Walsworth Publishing Co, 2000;923-936.
6. Rollin BE. Updating veterinary medical ethics. J Am Vet Med Assoc 1978;173:1015-1018

7. Lindell EM. Diagnosis and treatment of destructive behavior in dogs. Vet Clin North Am Small Anim Pract 1997;27:533-547.

8. Katz J. Reflections on informed consent: 40 years after its birth. J Am Coll Surg 1998;186:466-474.

9. Fontanarosa PB, Lundberg GD. Alternative medicine meets science. J Am Med Assoc 1998;280:1618-1619.

10. Sandler AD, Brazdziunas D, Cooley WC, et al. Committee on children with disabilities of the American Academy of Pediatrics policy report. Counseling families who choose complementary and alternative medicine for their child with chronic illness or disability. Pediatrics 2001;107:598-601.

11. Rollin BE. The use and abuse of Aesculapian authority in veterinary medicine. J Am Vet Med Assoc 2002;220:1144-1149.

12. Ubel PA. "What should I do, Doc?" Some psychological benefits of physician recommendations. Arch Int Med 2002;162: 977-980.

13. Gillis CL. Tendinitis (bowed tendon). In: Smith BP, ed. Large animal internal medicine. 2nd ed. St Louis: Mosby Year Book Inc, $1996 ; 1326-1328$. 